Article

\title{
Impact of Hyperbaric Oxygen Therapy on Subsequent Neurological Sequelae Following Carbon Monoxide Poisoning
}

\author{
Chien-Cheng Huang ${ }^{1,2,3}{ }^{-0}$, Chung-Han Ho ${ }^{4,5}$, Yi-Chen Chen ${ }^{4}$, Chien-Chin Hsu ${ }^{1,6}$, \\ Yi-Fong Wang ${ }^{7}$, Hung-Jung Lin ${ }^{1,6,8}$, Jhi-Joung Wang ${ }^{4}$ and How-Ran Guo ${ }^{2,9,10, *(1)}$ \\ 1 Department of Emergency Medicine, Chi-Mei Medical Center, Tainan 710, Taiwan; \\ chienchenghuang@yahoo.com.tw (C.-C.H.); nych2525@gmail.com (C.-C.H.); hjlin52@gmail.com (H.-J.L.) \\ 2 Department of Environmental and Occupational Health, College of Medicine, National Cheng Kung \\ University, Tainan 701, Taiwan \\ 3 Department of Senior Services, Southern Taiwan University of Science and Technology, Tainan 71005, Taiwan \\ 4 Department of Medical Research, Chi Mei Medical Center, Tainan 710, Taiwan; \\ ho.c.hank@gmail.com (C.-H.H.); laura751111986@hotmail.com (Y.-C.C.); 400002@mail.chimei.org.tw (J.-J.W.) \\ 5 Department of Hospital and Health Care Administration, Chia Nan University of Pharmacy and Science, \\ Tainan 71710, Taiwan \\ 6 Department of Biotechnology, Southern Taiwan University of Science and Technology, Tainan 71005, Taiwan \\ 7 Department of Leisure, Recreation and Tourism Management, Southern Taiwan University of Science and \\ Technology, Tainan 71005, Taiwan; yfwang@stust.edu.tw \\ 8 Department of Emergency Medicine, Taipei Medical University, Taipei 110, Taiwan \\ 9 Department of Occupational and Environmental Medicine, National Cheng Kung University Hospital, \\ Tainan 704, Taiwan \\ 10 Occupational Safety, Health, and Health Research Center, National Cheng Kung University Hospital, \\ Tainan 704, Taiwan \\ * Correspondence: hrguo@mail.ncku.edu.tw; Tel.: +886-6-235-3535
}

Received: 18 September 2018; Accepted: 11 October 2018; Published: 13 October 2018

\begin{abstract}
The purpose of this study was to evaluate the effects of hyperbaric oxygen therapy (HBOT) on reducing neurological sequelae (NS) in patients with carbon monoxide poisoning (COP). Using a nationwide database of insurance claims in Taiwan, we conducted a population-based cohort study to identify 24,046 patients with COP diagnosed between 1999 and 2012, including 6793 (28.2\%) patients who received HBOT and 17,253 (71.8\%) patients who did not. We followed the two cohorts of patients and compared the occurrence of NS. The two cohorts had similar sex ratios, but patients who received HBOT were younger $(34.8 \pm 14.8$ vs. $36.1 \pm 17.2$ years, $p<0.001)$. Patients who received HBOT had a higher risk for NS (adjusted hazard ratio [AHR]: 1.4; 95\% confidence interval [CI]: 1.4-1.5), after adjusting for age, sex, underlying comorbidities (hypertension, diabetes, chronic obstructive pulmonary disease, hyperlipidemia, malignancy, coronary artery disease, congestive heart failure, liver disease, renal disease, connective tissue disease, human immunodeficiency virus [HIV] infection, and alcoholism), monthly income, suicide, drug poisoning, and acute respiratory failure. We observed similar findings when we stratified the patients by age, sex, underlying comorbidities, and monthly income. The increased risk was most prominent in the first 2 weeks (AHR: 2.4; 95\% CI: 2.1-2.7) and remained significant up to 6 months later (AHR: 1.6; 95\% CI: 1.4-1.7). The risk for NS was higher in patients with COP who received HBOT than in those who did not, even after considering the possible impact of longer observation periods on survivors. Further studies that included the potential confounding factors we did not measure are needed to confirm findings in this study.
\end{abstract}

Keywords: carbon monoxide poisoning; hyperbaric oxygen therapy; neurological sequelae 


\section{Introduction}

Carbon monoxide (CO) is a toxic product from the incomplete combustion of carbon-based compounds by water heaters, charcoal burning, motor vehicles, gas-powered furnaces, and other domestic or industrial fuel-burning processes [1-3]. Even a small amount of CO can cause severe tissue hypoxia because the affinity of $\mathrm{CO}$ for hemoglobin is about 250 times greater than that for oxygen [4]. In the United States, carbon monoxide poisoning (COP) is responsible for an estimated 50,000 emergency department visits annually and is one of the leading causes of death due to poisoning $[5,6]$. In addition to accidental poisoning, COP by burning charcoal has become one of the most popular methods for suicide in recent years [7]. In Taiwan, the incidence of suicidal COP by burning charcoal increased by 25-fold between 1999 and 2009 [7].

The suggested treatment for COP is administration of $100 \%$ normobaricoxygen (NBO) by non-rebreathing mask or endotracheal intubation $[6,8]$. The additional benefit of hyperbaric oxygen therapy (HBOT) for reducing subsequent neurological sequelae (NS) has been a subject of debates [6,9-11]. A double-blind randomized trial in 2002 reported that HBOT can reduce the risk for NS at 6 weeks and 12 months, which is the most important evidence supporting HBOT [12]. However, a meta-analysis in 2011 showed that existing randomized trials failed to prove HBOT's effect of reducing NS [9]. On the other hand, our previous study found that HBOT was associated with a substantial reduction in the mortality after COP [13]. Therefore, we conducted a nationwide population-based cohort study in Taiwan to clarify this issue.

\section{Experimental Section}

\subsection{Data Source}

We used the Nationwide Poisoning Database (NPD), which is a sub-database from the Taiwan National Health Insurance Research Database (NHIRD), for this study. The NPD contains all the poisoning cases, including COP, between 1999 and 2013 in Taiwan. The NHIRD encompasses data from the Taiwan National Health Insurance program, which comprises nearly $100 \%$ of the population including foreigners in this country [14]. The database of this program contains registration files and original claim data for reimbursement [14]. Large computerized databases derived from this system by the National Health Insurance Administration, Ministry of Health and Welfare, Taiwan, and maintained by the National Health Research Institutes, Taiwan, are provided to scientists in Taiwan for research purposes [14].

\subsection{Identification of Patients with COP}

We identified all the patients with COP by the International Classification of Diseases, Ninth Revision, Clinical Modification (ICD-9-CM): 986, E868, E952, or E982 in either hospitalization or emergency department care as one of the main diagnoses from the NPD between 1999 and 2012 (Figure 1). The diagnosis of COP in Taiwan is generally based on documented CO exposure (elevated carboxyhemoglobin $[\mathrm{COHb}]$ levels or ambient $\mathrm{CO}$ concentrations) and any of the following symptoms: headache, malaise, fatigue, forgetfulness, dizziness, loss of consciousness, confusion, visual disturbances, nausea, vomiting, cardiac ischemia, or metabolic acidosis (a calculated base excess level $<-2.0 \mathrm{mmol} / \mathrm{L}$ or a blood lactate level $>2.5 \mathrm{mmol} / \mathrm{L}$ ). If the $\mathrm{COHb}$ level was $<10 \%$, the patient was eligible only if COP was the only plausible diagnosis $[3,4,15,16]$. Regarding NS, we focused on any manifestations of impairments in concentration, language, or calculation, cognitive dysfunction, delirium, amnesia, Parkinson-like syndromes, gait disturbance, corticospinal tract signs of damage, urine and fecal incontinence, vestibular problems, depression, anxiety, or affective changes [15]. Therefore, we defined the criteria of NS as including the diagnosis of degenerative diseases of the central nervous system (ICD-9-CM: 290, 331-337), psychiatric diseases (ICD-9-CM: 293-298, 300), or other diseases of the nervous system (ICD-9-CM: 338-352) in at least one hospitalization or three occurrences of ambulatory care after COP. Because the claim diagnosis may be incorrect in the ambulatory care, we used at 
least three occurrences of ambulatory care to confirm the diagnosis, which has been widely used and confirmed in studies based on the NHIRD [17]. Patients who had the same diagnosis of neurological or psychiatric diseases before and after COP were excluded to ensure that the onset of NS was after the poisoning.

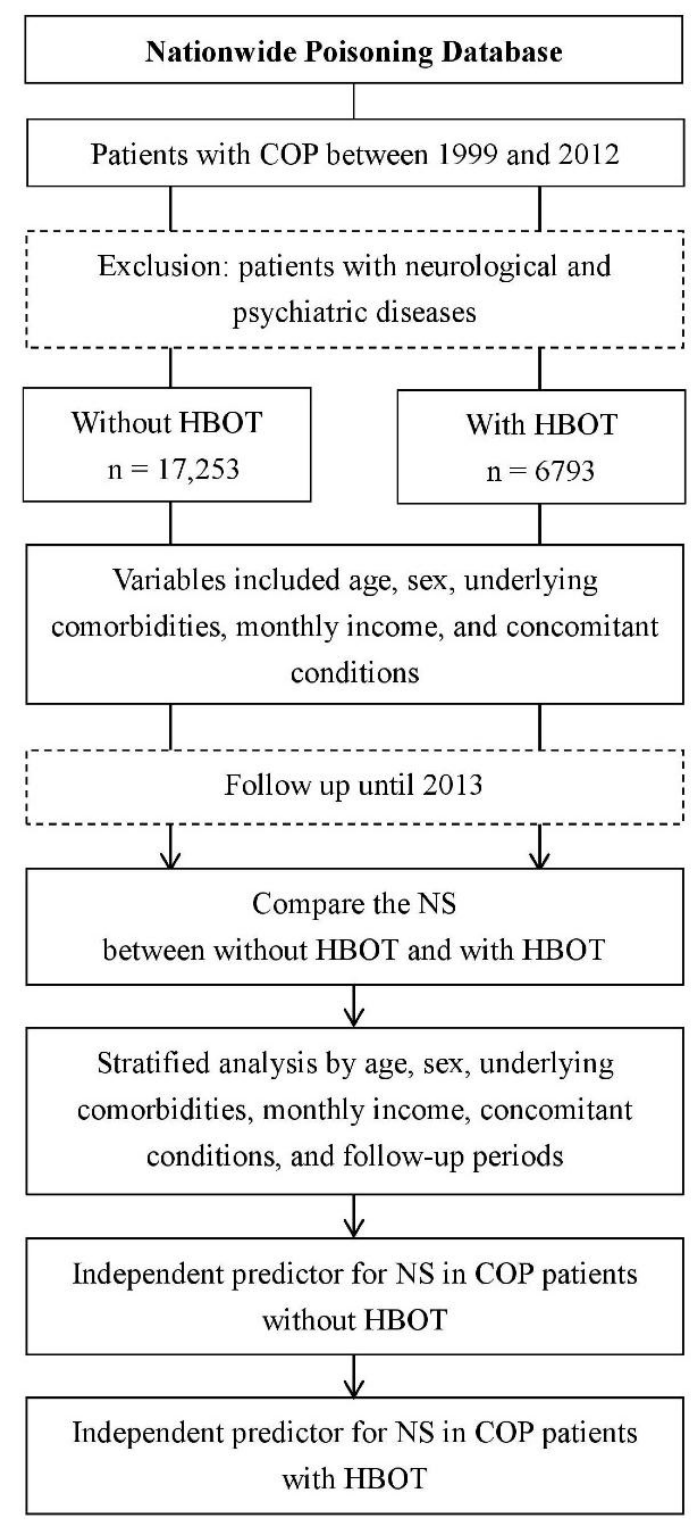

Figure 1. Flowchart of this study. COP, carbon monoxide poisoning; HBOT, hyperbaric oxygen therapy; NS, neurological sequelae.

\subsection{Definitions of Variables}

In addition to COP, we included the following variables in the analysis: age, sex, underlying comorbidities, monthly income, and concomitant conditions. Age was classified into the following subgroups: <20, 20-34, 35-49, 50-64, and $\geq 65$ years. Underlying comorbidities were defined as at least one hospitalization or three occurrences of ambulatory care of the diagnoses before COP: hypertension (ICD-9-CM 401-405), diabetes (ICD-9-CM 250), chronic obstructive pulmonary disease (COPD; ICD-9-CM 496), hyperlipidemia (ICD-9-CM 272), malignancy (ICD-9-CM 140-208), coronary artery disease (ICD-9-CM 410-414), congestive heart failure (ICD-9-CM 428), liver disease (ICD-9-CM 570-576), renal disease (ICD-9-CM 580-593), connective tissue disease (ICD-9-CM 710), HIV infection (ICD-9-CM 042, 079.53, V08), and alcoholism (ICD-9-CM 291, 303, 3050, 3575, 4255, 5353, 
5710-5713, or V113). Monthly income was classified as $<19,999,20,000-39,999$, and $\geq 40,000$ New Taiwan Dollars (NTD) [18]. Concomitant conditions were defined as suicide (management codes 94.0 or 94.1, or ICD-9-CM E950-E959), drug poisoning (ICD-9-CM 960-989, exclusion of 986), acute respiratory failure (ICD-9-CM 518.81 or 518.84, or management codes 960, 9601, 9602, 9603, 9604, 9605, 9390, 9391, or 311), acute myocardial injury (ICD-9-CM 410), acute hepatitis (ICD-9-CM 573.3), and acute renal failure (ICD-9-CM 584 or management code 339.5) during the same episode of COP. The references for ICD-9-CM and management code can be found in the websites of Taiwan National Health Insurance Administration $[19,20]$.

\subsection{Comparison of the Risk for NS Between the Two Cohorts and Independent Predictors for NS}

We compared the risk for NS between the two cohorts until 2013. Stratified analyses by age, sex, underlying comorbidities, monthly income, suicide, drug poisoning, acute respiratory failure, and follow-up periods were also performed to evaluate the presence of effect modification. The independent predictors for NS in the two cohorts were also investigated individually.

\subsection{Ethics Statement}

The Institutional Review Board at Chi-Mei Medical Center approved this study, which was conducted in strict accordance with the Declaration of Helsinki. Given that the NPD contains de-identified information, the need for informed consent from the patients was waived as it did not affect the rights or welfare of the patients.

\subsection{Data Analysis}

Independent $\mathrm{t}$-tests for continuous variables and chi-square tests for categorical variables were used to evaluate the differences in demographic characteristics, underlying comorbidities, monthly income, and concomitant conditions between the two cohorts. Cox proportional hazard regression and Kaplan-Meier method with log-rank test were performed to compare the incidence of NS between the two cohorts. We performed additional Poisson regression analysis to evaluate the risk for NS stratified by follow-up periods between the two cohorts. Cox proportional hazard regression analysis was conducted to investigate the independent predictors for NS in the two cohorts individually. We also used propensity score matching and Cox proportional hazard regression analysis to validate the result. Because a longer survival period leads to a higher probability of developing NS, we conducted an additional analysis combining NS and death as the outcome to evaluate the possible effect. All the analyses were performed using SAS 9.4 for Windows (SAS Institute, Cary, NC, USA) at a two-tailed significance level of 0.05 .

\section{Results}

A total of 24,046 COP patients, including 6793 (28.2\%) patients who received HBOT and 17,253 $(71.8 \%)$ patients who did not, were identified in this study (Table 1). Patients who received HBOT were younger than those who did not $(34.8 \pm 14.8$ years vs. $36.1 \pm 17.2$ years, $p<0.001)$. The sex ratio and monthly income did not differ between the two cohorts. Patients who received HBOT had lower prevalence of underlying comorbidities of hypertension, diabetes, hyperlipidemia, malignancy, coronary artery disease, congestive heart failure, COPD, liver disease, and alcoholism, but not other comorbidities. During the COP episode, patients who received HBOT presented higher percentages of suicide (31.2\% vs. $15.3 \%)$, acute respiratory failure $(9.7 \%$ vs. $6.2 \%)$, and acute renal failure $(1.9 \%$ vs. $1.0 \%)$ than the patients who did not. 
Table 1. Demographic characteristics, underlying comorbidities, monthly income, and concomitant conditions in the patients with COP between 1999 and 2012.

\begin{tabular}{|c|c|c|c|c|}
\hline Variable & Total Patients $n=24,046(100.0)$ & Without HBOT $n=17,253$ (71.8) & With HBOT $n=6793$ (28.2) & $p$-Value * \\
\hline Age (years) & $35.7 \pm 16.6$ & $36.1 \pm 17.2$ & $34.8 \pm 14.8$ & $<0.001$ \\
\hline \multicolumn{5}{|l|}{ Age subgroup (years) } \\
\hline$<20$ & $3382(14.1)$ & $2509(14.5)$ & $873(12.9)$ & $<0.001$ \\
\hline $20-34$ & $8987(37.4)$ & $6221(36.1)$ & $2766(40.7)$ & \\
\hline $35-50$ & $7293(30.3)$ & $5148(29.8)$ & $2145(31.6)$ & \\
\hline $51-64$ & $2996(12.5)$ & $2223(12.9)$ & $773(11.4)$ & \\
\hline$\geq 65$ & $1388(5.8)$ & $1152(6.7)$ & $236(3.5)$ & \\
\hline \multicolumn{5}{|l|}{ Sex } \\
\hline Female & $12,062(50.2)$ & $8653(50.2)$ & $3409(50.2)$ & 0.966 \\
\hline Male & $11,984(49.8)$ & $8600(49.9)$ & $3384(49.8)$ & \\
\hline \multicolumn{5}{|l|}{ Underlying comorbidity } \\
\hline Hypertension & $2815(11.7)$ & $2216(12.8)$ & $599(8.8)$ & $<0.001$ \\
\hline Diabetes & $1499(6.2)$ & $1137(6.6)$ & $362(5.3)$ & $<0.001$ \\
\hline Chronic obstructive pulmonary disease & $385(1.6)$ & $320(1.9)$ & $65(1.0)$ & $<0.001$ \\
\hline Hyperlipidemia & $1967(8.2)$ & $1486(8.6)$ & $481(7.1)$ & $<0.001$ \\
\hline Malignancy & $643(2.7)$ & $499(2.9)$ & $144(2.1)$ & $<0.001$ \\
\hline Coronary artery disease & $1358(5.7)$ & $1067(6.2)$ & $291(4.3)$ & $<0.001$ \\
\hline Congestive heart failure & $392(1.6)$ & $326(1.9)$ & $66(1.0)$ & $<0.001$ \\
\hline Liver disease & $3307(13.8)$ & $2428(14.1)$ & $879(12.9)$ & 0.022 \\
\hline Renal disease & $2445(10.2)$ & $1804(10.5)$ & $641(9.4)$ & 0.013 \\
\hline Connective tissue disease & $208(0.9)$ & $146(0.9)$ & $62(0.9)$ & 0.616 \\
\hline HIV infection & $61(0.3)$ & $46(0.3)$ & $15(0.2)$ & 0.525 \\
\hline Alcoholism & $861(3.6)$ & $668(3.9)$ & $193(2.8)$ & $<0.001$ \\
\hline \multicolumn{5}{|l|}{ Monthly income (NTD) } \\
\hline$<19,999$ & $17,622(73.3)$ & $12,670(73.4)$ & $4952(72.9)$ & 0.156 \\
\hline $20,000-39,999$ & $5122(21.3)$ & $3629(21.0)$ & $1493(22.0)$ & \\
\hline$\geq 40,000$ & $1302(5.4)$ & $954(5.5)$ & $348(5.1)$ & \\
\hline \multicolumn{5}{|l|}{ Concomitant condition } \\
\hline Suicide & $4763(19.8)$ & $2642(15.3)$ & $2121(31.2)$ & $<0.001$ \\
\hline Drug poisoning & $245(1.0)$ & $187(1.1)$ & $58(0.9)$ & 0.110 \\
\hline Acute respiratory failure & $1737(7.2)$ & $1077(6.2)$ & $660(9.7)$ & $<0.001$ \\
\hline Acute myocardial injury & $54(0.2)$ & $39(0.2)$ & $15(0.2)$ & 0.939 \\
\hline Acute hepatitis & $52(0.2)$ & $39(0.2)$ & $13(0.2)$ & 0.602 \\
\hline Acute renal failure & $299(1.2)$ & $167(1.0)$ & $132(1.9)$ & $<0.001$ \\
\hline
\end{tabular}


Cox proportional hazard regression analysis showed a higher risk for NS among the patients who received HBOT than the patients who did not (adjusted hazard ratio [AHR]: 1.4; $95 \%$ confidence interval [CI]: 1.4-1.5) after adjusting for age, sex, monthly income, suicide, drug poisoning, acute respiratory failure and underlying comorbidity of hypertension, diabetes, COPD, hyperlipidemia, malignancy, coronary artery disease, congestive heart failure, liver disease, renal disease, connective tissue disease, HIV infection, and alcoholism (Table 2). Stratified analyses showed that the increased risk for NS in the patients who received HBOT was also significant in the five age subgroups, two sex subgroups, all subgroups defined by the underlying comorbidities except for congestive heart failure, three subgroups of monthly income, and subgroups defined by concomitant conditions of suicide, drug poisoning, and acute respiratory failure. During the follow-up period, patients who received HBOT presented the highest risk for NS in the first 2 weeks after COP (AHR: 2.4; 95\% CI: 2.1-2.7), followed by $2-4$ weeks (AHR: 1.6 ; 95\% CI: 1.4-1.9) and 1-6 months (AHR: 1.6 ; 95\% CI: 1.4-1.7). Poisson regression analysis showed similar findings: the incidence rate ratio (IRR) was the highest in 2 weeks (2.6; 95\% CI: 2.3-2.9), followed by $2-4$ weeks (IRR: 2.3 ; 95\% CI: $2.0-2.7$ ) and 1-6 months (IRR: 1.8 ; 95\% CI: 1.7-2.0). Kaplan-Meier method with log-rank test also demonstrated a higher risk for NS in the patients who received HBOT than in the patients who did not $(p<0.0001$; Figure 2$)$. Propensity score matching and Cox proportional hazard regression analysis also showed patients who received HBOT had a higher risk for NS than those without (AHR: 1.4; 95\% CI: 1.3-1.5) (Supplement Tables S1 and S2). In the additional analysis combining NS and death as the outcome, patients who received HBOT still had a higher risk than those who did not, but the difference became smaller (AHR: 1.2; 95\% CI: 1.1-1.2).

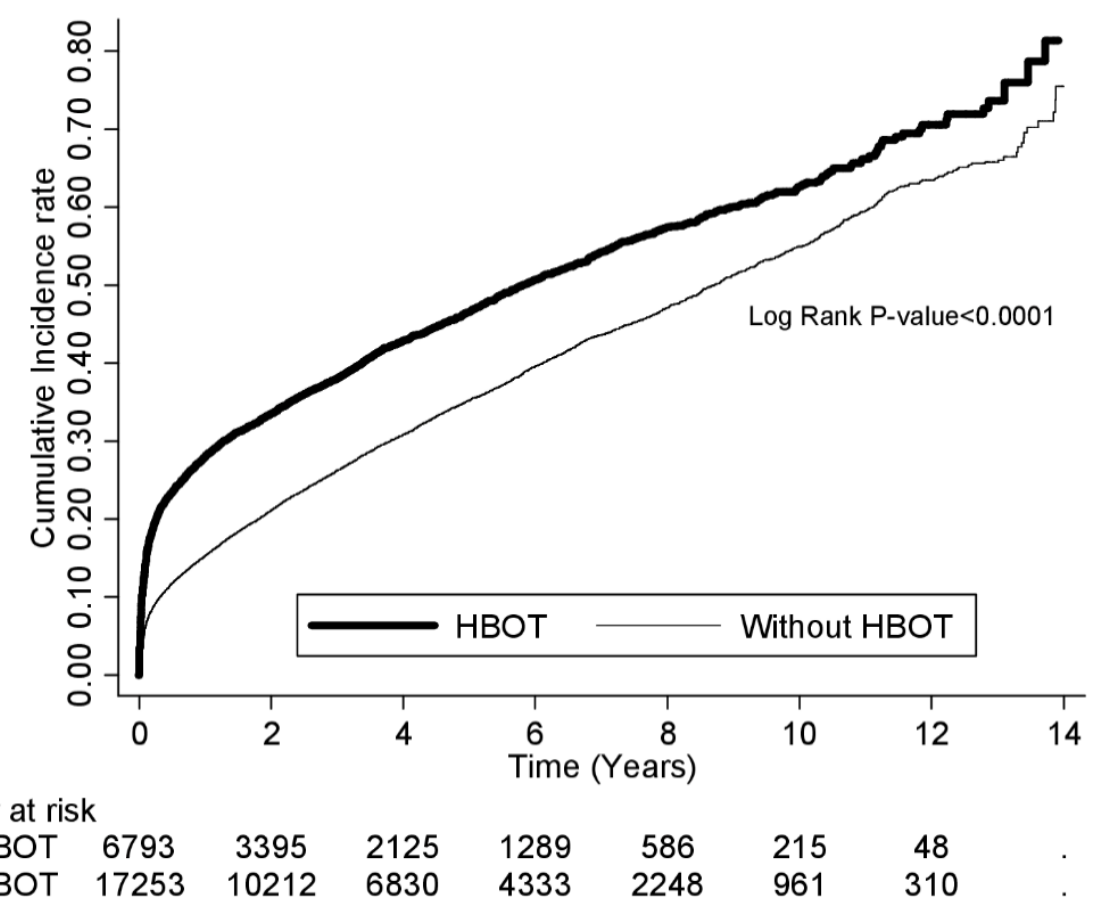

Figure 2. Follow-up risk for NS between COP patients with and without HBOT by Kaplan-Meier method with log-rank test. 
Table 2. Comparison of the risk for NS between patients with COP who received HBOT and who did not by Cox proportional hazard regression and Poisson regression analyses.

\begin{tabular}{|c|c|c|c|c|c|c|c|c|c|}
\hline \multirow{2}{*}{ Variable } & \multicolumn{3}{|c|}{ With НBOT } & \multicolumn{3}{|c|}{ Without HBOT (reference) } & \multirow{2}{*}{ Crude HR $(95 \%$ CI) } & \multirow{2}{*}{ AHR $(95 \% \mathrm{CI}) *$} & \multirow{2}{*}{$\operatorname{IRR}(95 \% \mathrm{CI})$} \\
\hline & Case & PY & Rate & Case & PY & Rate & & & \\
\hline Overall analysis & 3043 & $20,765.3$ & 146.5 & 5992 & $64,832.7$ & 92.4 & $1.5(1.4-1.6)$ & $1.4(1.4-1.5)$ & - \\
\hline \multicolumn{10}{|l|}{ Stratified analysis } \\
\hline $\begin{array}{c}\text { Degenerative diseases of the central } \\
\text { nervous system }\end{array}$ & 657 & $28,291.9$ & 23.2 & 1197 & $80,595.3$ & 14.9 & $1.5(1.3-1.6)$ & $1.5(1.3-1.6)$ & - \\
\hline Psychiatric diseases & 2076 & $23,642.8$ & 87.8 & 4145 & $69,828.3$ & 59.3 & $1.4(1.3-1.5)$ & $1.3(1.2-1.4)$ & - \\
\hline \multicolumn{10}{|l|}{ Age (years) } \\
\hline$<20$ & 217 & 3851.3 & 56.3 & 465 & $12,736.9$ & 36.5 & $1.5(13-1.8)$ & $1.4(1.2-1.7)$ & - \\
\hline $20-34$ & 1243 & 8449.4 & 147.1 & 2170 & $24,724.1$ & 87.8 & $1.6(1.5-1.7)$ & $1.4(1.3-1.5)$ & - \\
\hline $35-50$ & 1060 & 6093.6 & 174.0 & 1999 & $18,418.8$ & 108.5 & $1.5(1.4-1.6)$ & $1.4(1.3-1.5)$ & - \\
\hline $51-64$ & 393 & 1890.4 & 207.9 & 871 & 6226.9 & 139.9 & $1.5(1.3-1.6)$ & $1.4(1.3-1.6)$ & - \\
\hline $\begin{array}{l}\geq 65 \\
\text { Sex }\end{array}$ & 130 & 480.6 & 270.5 & 487 & 2726.2 & 178.6 & $1.5(1.2-1.8)$ & $1.4(1.2-1.7)$ & - \\
\hline Female & 1566 & $10,574.5$ & 148.1 & 3235 & $33,324.4$ & 97.1 & $1.5(1.4-1.6)$ & $1.6(1.3-1.4)$ & - \\
\hline Male & 1477 & $10,190.8$ & 144.9 & 2757 & $31,508.3$ & 87.5 & $1.6(1.5-1.7)$ & $1.5(1.4-1.6)$ & - \\
\hline \multicolumn{10}{|l|}{ Underlying comorbidity } \\
\hline Hypertension & 309 & 1374.7 & 224.8 & 925 & 5416.5 & 170.8 & $1.3(1.1-1.5)$ & $1.3(1.1-1.5)$ & - \\
\hline Diabetes & 167 & 852.4 & 195.9 & 437 & 2676.5 & 163.3 & $1.2(1.0-1.4)$ & $1.2(1.0-1.4)$ & - \\
\hline Chronic obstructive pulmonary disease & 42 & 104.8 & 400.7 & 129 & 730.3 & 176.7 & $2.1(1.4-2.9)$ & $2.1(1.4-3.0)$ & - \\
\hline Hyperlipidemia & 257 & 1008.0 & 255.0 & 577 & 3700.7 & 155.9 & $1.6(1.4-1.8)$ & $1.5(1.3-1.8)$ & - \\
\hline Malignancy & 72 & 258.7 & 278.3 & 184 & 989.0 & 186.1 & $1.4(1.1-1.9)$ & $1.4(1.1-1.9)$ & - \\
\hline Coronary artery disease & 148 & 621.3 & 238.2 & 452 & 2490.7 & 181.5 & $1.3(1.1-1.6)$ & $1.3(1.0-1.5)$ & - \\
\hline Congestive heart failure & 33 & 130.6 & 252.7 & 122 & 616.9 & 197.8 & $1.3(0.9-1.9)$ & $1.5(1.0-2.3)$ & - \\
\hline Liver disease & 446 & 2059.1 & 216.6 & 1037 & 6424.1 & 161.4 & $1.3(1.2-1.5)$ & $1.3(1.1-1.4)$ & - \\
\hline Renal disease & 319 & 1490.7 & 214 & 707 & 4975.9 & 142.1 & $1.5(1.3-1.7)$ & $1.4(1.2-1.6)$ & - \\
\hline Connective tissue disease & 38 & 121.9 & 311.7 & 55 & 345.1 & 159.4 & $1.9(1.3-2.9)$ & $2.0(1.2-3.2)$ & - \\
\hline HIV infection & 9 & 29.2 & 308.3 & 13 & 118.5 & 109.7 & $2.5(1.1-5.9)$ & $10.3(2.3-45.8)$ & - \\
\hline Alcoholism & 115 & 381.3 & 301.6 & 308 & 1574.3 & 195.7 & $1.5(1.2-1.9)$ & $1.6(1.2-1.9)$ & - \\
\hline \multicolumn{10}{|l|}{ Monthly income (NTD) } \\
\hline$<19,999$ & 2322 & $15,060.2$ & 154.2 & 4573 & $47,295.3$ & 96.7 & $1.5(1.4-1.6)$ & $1.4(1.4-1.5)$ & - \\
\hline $20,000-39,999$ & 586 & 4516.8 & 129.7 & 1142 & $13,501.0$ & 84.6 & $1.5(1.3-1.6)$ & $1.4(1.2-1.5)$ & - \\
\hline$\geq 40,000$ & 135 & 1188.4 & 113.6 & 277 & 4036.4 & 68.6 & $1.6(1.3-1.9)$ & $1.5(1.2-1.9)$ & - \\
\hline
\end{tabular}


Table 2. Cont.

\begin{tabular}{|c|c|c|c|c|c|c|c|c|c|}
\hline \multirow{2}{*}{ Variable } & \multicolumn{3}{|c|}{ With HBOT } & \multicolumn{3}{|c|}{ Without HBOT (reference) } & \multirow{2}{*}{ Crude HR $(95 \%$ CI) } & \multirow{2}{*}{$\operatorname{AHR}(95 \% \mathrm{CI}) *$} & \multirow{2}{*}{ IRR $(95 \%$ CI $)$} \\
\hline & Case & PY & Rate & Case & PY & Rate & & & \\
\hline \multicolumn{10}{|l|}{ Suicide } \\
\hline Yes & 1181 & 5664.8 & 208.5 & 1305 & 8639.4 & 151.1 & $1.3(1.2-1.4)$ & $1.3(1.2-1.4)$ & - \\
\hline No & 1862 & $15,100.5$ & 123.3 & 4687 & $56,193.3$ & 83.4 & $1.4(1.3-1.5)$ & $1.5(1.4-1.5)$ & - \\
\hline \multicolumn{10}{|l|}{ Drug poisoning } \\
\hline Yes & 33 & 116.0 & 284.4 & 81 & 620.2 & 130.6 & $1.9(1.2-2.8)$ & $1.6(1.0-2.6)$ & - \\
\hline No & 3010 & $20,649.3$ & 145.8 & 5911 & $64,212.5$ & 92.1 & $1.5(1.4-1.6)$ & $1.4(1.4-1.5)$ & - \\
\hline \multicolumn{10}{|c|}{ Acute respiratory failure } \\
\hline Yes & 429 & 1268.5 & 338.2 & 440 & 2281.6 & 192.9 & $1.6(1.4-1.8)$ & $1.5(1.3-1.8)$ & - \\
\hline No & 2614 & $19,496.8$ & 134.1 & 5552 & $62,551.1$ & 88.8 & $1.4(1.4-1.5)$ & $1.4(1.3-1.5)$ & - \\
\hline \multicolumn{10}{|c|}{ Follow-up period } \\
\hline$<2$ weeks & 597 & 245.2 & 2434.7 & 579 & 620.5 & 933.1 & $2.6(2.3-2.9)$ & $2.4(2.1-2.7)$ & $2.6(2.3-2.9)$ \\
\hline 2-4 weeks & 283 & 290.6 & 973.8 & 308 & 733.9 & 419.7 & $1.7(1.5-2.0)$ & $1.6(1.4-1.9)$ & $2.3(2.0-2.7)$ \\
\hline $1-6$ months & 682 & 2279.1 & 299.2 & 1010 & 6162.6 & 163.9 & $1.8(1.6-2.0)$ & $1.6(1.4-1.7)$ & $1.8(1.7-2.0)$ \\
\hline 6-12 months & 283 & 2871.4 & 98.6 & 537 & 7661.6 & 70.1 & $1.7(0.9-1.2)$ & $1.0(0.8-1.1)$ & $1.4(1.2-1.6)$ \\
\hline $1-2$ years & 306 & 5422.4 & 56.4 & 816 & $15,028.2$ & 54.3 & $1.0(0.9-1.2)$ & $0.9(0.8-1.1)$ & $1.0(0.9-1.2)$ \\
\hline $2-4$ years & 416 & $10,066.8$ & 41.3 & 1121 & $28,572.7$ & 39.2 & $1.0(0.9-1.1)$ & $0.9(0.8-1.1)$ & $1.1(0.9-1.2)$ \\
\hline$\geq 4$ years & 476 & $20,765.3$ & 22.9 & 1621 & $64,832.7$ & 25.0 & $1.1(0.9-1.2)$ & $1.0(0.9-1.2)$ & $0.9(0.8-1.0)$ \\
\hline
\end{tabular}

NS, neurological sequelae; COP, carbon monoxide poisoning; HBOT, hyperbaric oxygen therapy; HR, hazard ratio; AHR, adjusted hazard ratio; CI, confidence interval; IRR, incidence rate ratio; PY, person-years; Rate, NS rate per 1000 person-years; NTD, new Taiwan dollars. * Adjusted for age, sex, underlying comorbidity of hypertension, diabetes, hyperlipidemia, malignancy, coronary artery disease, congestive heart failure, chronic obstructive pulmonary disease, liver disease, renal disease, connective tissue disease, HIV infection, alcoholism, monthly income, suicide, drug poisoning, and acute respiratory failure. 
The incidence rates of degenerative diseases of the central nervous system, psychiatric diseases, and other diseases of the nervous system were $23.2,87.8$, and 57.6 per 1000 person-years in patients who received HBOT and 14.9, 59.3, and 34.9 per 1000 person-years in patients who did not receive HBOT, respectively (Table 2). The AHR observed in the analysis of each subgroup of NS was similar to that observed in the overall analysis.

The independent predictors for NS in the patients who received HBOT were old age; male sex; low monthly income; underlying comorbidity of hypertension, malignancy, liver disease, and alcoholism; and concomitant conditions of suicide and acute respiratory failure (Table 3). Except for comorbidity of hypertension, malignancy, and liver disease, they are also independent predictors for NS in the patients who did not receive HBOT (Table 3). However, underlying comorbidity of COPD and hyperlipidemia also appeared to be independent predictors for NS in patients who did not receive HBOT.

Table 3. Independent predictors for NS in patients with COP by Cox proportional hazard regression analysis.

\begin{tabular}{|c|c|c|c|c|}
\hline Variable & \multicolumn{2}{|c|}{ Patients with COP Who Received HBOT } & \multicolumn{2}{|c|}{ Patients with COP Who Did Not Receive HBOT } \\
\hline \multicolumn{5}{|l|}{ Age (years) } \\
\hline $20-34$ & $2.4(2.1-2.6)$ & $2.3(2.1-2.5)$ & $2.3(2.0-2.7)$ & $2.2(1.9-2.5)$ \\
\hline $35-50$ & $2.9(2.6-3.2)$ & $2.6(2.3-2.9)$ & $2.7(2.3-3.1)$ & $2.4(2.0-2.8)$ \\
\hline $51-64$ & $3.5(3.1-3.9)$ & $2.8(2.5-3.2)$ & $3.0(2.5-3.5)$ & $2.6(2.2-3.1)$ \\
\hline Female & 1 & 1 & 1 & 1 \\
\hline Male & $0.9(0.9-0.9)$ & $1.2(1.2-1.3)$ & $1.0(0.9-1.1)$ & $1.1(1.0-1.2)$ \\
\hline \multicolumn{5}{|l|}{ Monthly income (NTD) } \\
\hline$<19,999$ & $1.4(1.2-1.6)$ & $1.4(1.3-1.6)$ & $1.3(1.1-1.6)$ & $1.3(1.1-1.6)$ \\
\hline $20,000-39,999$ & $1.2(1.1-1.4)$ & $1.2(1.0-1.3)$ & $1.1(0.9-1.3)$ & $1.1(0.9-1.3)$ \\
\hline $\begin{array}{l}\text { Chronic obstructive } \\
\text { pulmonary disease }\end{array}$ & $1.7(1.4-2.0)$ & $1.0(0.8-1.2)$ & $2.0(1.5-2.7)$ & $1.5(1.1-2.1)$ \\
\hline Hyperlipidemia & $1.6(1.5-1.7)$ & $1.0(0.9-1.1)$ & $1.5(1.3-1.7)$ & $1.3(1.1-1.5)$ \\
\hline Malignancy & $1.8(1.5-2.0)$ & $1.2(1.0-1.4)$ & $1.5(1.2-1.9)$ & $1.1(0.9-1.4)$ \\
\hline Coronary artery disease & $1.8(1.7-2.0)$ & $1.1(1.0-1.2)$ & $1.4(1.2-1.6)$ & $1.0(0.8-1.2)$ \\
\hline Congestive heart failure & $1.8(1.5-2.1)$ & $1.0(0.8-1.2)$ & $1.4(1.0-2.0)$ & $0.9(0.6-1.3)$ \\
\hline Liver disease & $1.8(1.6-1.9)$ & $1.3(1.2-1.4)$ & $1.4(1.2-1.5)$ & $1.1(0.9-1.2)$ \\
\hline Renal disease & $1.5(1.4-1.6)$ & $1.1(1.0-1.2)$ & $1.3(1.2-1.5)$ & $1.1(1.0-1.2)$ \\
\hline Connective tissue disease & $1.6(1.2-2.1)$ & $1.1(0.9-1.5)$ & $1.7(1.3-2.4)$ & $1.3(1.0-1.8)$ \\
\hline HIV infection & $1.1(0.6-1.9)$ & $1.0(0.6-1.7)$ & $1.7(0.9-3.2)$ & $1.2(0.6-2.3)$ \\
\hline Alcoholism & $1.9(1.7-2.2)$ & $1.4(1.2-1.5)$ & $1.7(1.4-2.0)$ & $1.3(1.1-1.6)$ \\
\hline \multicolumn{5}{|l|}{ Concomitant condition } \\
\hline
\end{tabular}

NS, neurological sequelae; COP, carbon monoxide poisoning; HBOT, hyperbaric oxygen therapy; HR, hazard ratio; AHR, adjusted hazard ratio; CI, confidence interval; NTD, new Taiwan dollars. * Adjusted for age, sex, underlying comorbidity of hypertension, diabetes, hyperlipidemia, malignancy, coronary artery disease, congestive heart failure, chronic obstructive pulmonary disease, liver disease, renal disease, connective tissue disease, HIV infection, alcoholism, monthly income, suicide, drug poisoning, and acute respiratory failure.

\section{Discussion}

This study showed that the risk for NS in the patients who received HBOT was higher than that in the patients who did not, especially in the first 6 months after poisoning. The increased risk was also found in the subgroup analyses, including those by age, sex, underlying comorbidities except for congestive heart failure, monthly income, and concomitant conditions of suicide, drug poisoning, and acute respiratory failure. The common independent predictors for NS in the two cohorts were old age, male sex, low monthly income, underlying comorbidity of alcoholism, and concomitant conditions of suicide, and acute respiratory failure. 
A meta-analysis conducted in 2011 showed that existing randomized controlled trials reported conflicting results regarding HBOT vs. NBO [9]. A prospective randomized controlled trial recruiting 385 patients with acute domestic COP showed that HBOT had no benefit in patients with transient loss of consciousness and resulted in poor outcome in comatose patients [21]. The results might have been affected by the lack of blinding, loss to follow-up (at least 14\% at 1 month), and the difference in the proportion of coma cases before admission. Nevertheless, a double-blind randomized controlled trial reported that HBOT had no benefit and might even have worsened the outcome [22]. While one of our previous studies showed that COP was associated with increased long-term mortality [23], another showed that HBOT reduced long-term mortality [13]. Therefore, a possible explanation of the higher risk for NS in the patients who received HBOT was that the life-saving effect of HBOT is more prominent than its possible NS-reducing effect. In other words, HBOT might have saved the lives of some patients who were at higher risks for NS but would have passed away before developing NS if not for the HBOT. Our previous study found that HBOT was associated with an overall $36 \%$ reduction in the mortality (AHR: $0.74 ; 95 \%$ CI: $0.67-0.81$ ) and that the effect was most prominent in the first 2 weeks (AHR: 0.51 ; 95\% CI: 0.37-0.72) [13], which is also the period with the highest mortality in the current study. However, the additional analysis that combined NS and death as the outcome demonstrated that the AHR decreased to 1.2 but was still statistically significant (95\% CI: 1.1-1.2), indicating the presence of other factors. Another possible factor is that patients with a higher risk for NS were prone to receive HBOT (Table 1) in this study. The general indications for HBOT are also risk factors for NS [24-30], and even though we have adjusted for age and comorbidities in the analyses, we were unable to adjust for all the other risk factors. As a results, patients who received HBOT had higher prevalence of having risk factors for NS in comparison with those who did not, and this would contribute to the higher incidence of NS associated with HBOT observed in this study.

The protocol for HBOT differs among studies, which might affect the outcome. The most well-known protocol for HBOT is three hyperbaric oxygen treatments including 3 atmosphere absolute (ATA) at first chamber session and 2 ATA at second and third chamber sessions within a $24 \mathrm{~h}$ period [12]. This protocol reduced the risk for NS at 6 weeks and 12 months after acute COP [12]. However, the double-blind randomized controlled trial might suffer from bias introduced during data analysis. The potential sources of the bias included the differences in the outcomes analyzed between the preliminary report and the final report, reassigning participants to different groups based on assumptions, and the premature termination of the trial [9]. In Taiwan, the following protocol is generally adopted, but variations exist due to the differences in facilities and staff: (1) first session within 6-24 h as soon as possible; (2) 2.5-3.0 ATA; (3) 60-120 min each session; (4) treatments of oxygen supply (non-rebreathing mask vs. endotracheal intubation) and chamber choice (monoplace vs. multiplace chamber) depending on the patient's condition and medical resource; (5) one session each day; and (6) one to five sessions in total $[13,30]$. However, no studies have been conducted to compare the two protocols.

In our study, suicide was a predictor for NS in both patients who have received and not received HBOT, independent of other well-known risk factors. In the past 20 years, burning charcoal has become one of the most common methods of suicide in Taiwan and Hong Kong, contributing to a $20 \%$ increase in the overall suicide rate [31]. Patients attempting suicide might be more severely intoxicated, and thus might have worse prognosis. In the present study, low income predicted NS, which was similar to our previous finding that low income was an independent predictor of long-term mortality after COP [23]. Low income is associated with poor living environment and limited resources (such as CO detectors), which might contribute to the increased risk [32,33]. Furthermore, low income is associated with increased poor awareness of disease prevention, less and inconvenient access to medical care, and behavioral risk factors such as smoking [34,35], which might also contribute to this finding in the present study.

The independent predictors of NS differed between patients who have received and not received HBOT in our study. Specifically, while both COPD (AHR: 1.5; 95\% CI: 1.1-2.1) and hyperlipidemia 
(AHR: 1.3; 95\% CI: 1.1-1.5) were independent predictors of NS in patients who did not receive HBOT, they were associated with decreased AHRs (1.0(95\% CI: 0.8-1.2) for COPD and 1.0(95\% CI: 0.9-1.1) for hyperlipidemia) in patients who received HBOT.

This nationwide population-based study involved a large sample size to delineate a controversial issue in clinical practice. Nonetheless, our study had some limitations. First, due to the retrospective design of this study, some unmeasured confounders such as disease severity might have affected the result, although we had adjusted for all the potential confounders for which we were able to obtain data. Hospital-based studies adjusting for these unmeasured potential confounders are needed to confirm our findings. Second, NS was defined as a new-onset neurological or psychiatric disease after COP. This definition might overestimate the occurrence of NS and dilute the effect of real neurological damage sustained during the poisoning, but it might not affect our conclusions because it should lead to random (non-differential) misclassifications. Third, we did not evaluate the severity of NS because the claim data did not include information on severity. Further study may be necessary to clarify this issue. Fourth, we did not evaluate the association between the duration before HBOT was started after establishing the diagnosis of COP and the occurrence of NS because the claim data did not include information on such durations. Fifth, although this study covered the whole nation, it may not be generalized to other nations due to the difference in race, treatment protocol, and medical resources.

\section{Conclusions}

This nationwide population-based cohort study showed that the risk for NS was higher in the COP patients who received HBOT in comparison with those who did not. Because HBOT reduces mortality, the fact that high-risk survivors tended to develop NS afterwards might contribute to the increase in the risk. Another likely contributing factor was that patients who had risk factors for NS were more likely to receive HBOT. Further prospective studies that include potential confounding factors that we did not measure are warranted to confirm our speculations on the factors contributing to the increased risk for NS.

Supplementary Materials: The following are available online at http:/ / www.mdpi.com/2077-0383/7/10/349/s1, Table S1: Demographic characteristics, underlying comorbidities, monthly income, and concomitant conditions in the patients with COP between 1999 and 2012, Table S2: Comparison of the risk for NS between patients with COP who received HBOT and who did not by Cox proportional hazard regression analysis.

Author Contributions: C.-C.H. and H.-R.G. designed and conceived this study and wrote the first draft of the manuscript. H.-C.H. and Y.-C.C. performed the statistical analysis. C.-C.H., Y.-F.W., H.-J.L., and J.-J.W. provided professional suggestions during the study and interpretation of study results. All authors participated in the revision of the manuscript and approved the final manuscript.

Funding: This study was supported by Grant CMFHR10734 and CMFHR10753 from the Chi-Mei Medical Center, as well as the Headquarters of University Advancement at the National Cheng Kung University, which is supported by the Ministry of Education, Taiwan, R.O.C.

Conflicts of Interest: The authors declare no conflict of interest.

$\begin{array}{ll}\text { Abbreviations } \\ \text { HBOT } & \begin{array}{l}\text { hyperbaric oxygen therapy } \\ \text { neurological sequelae } \\ \text { carbon monoxide poisoning } \\ \text { COP }\end{array} \\ \text { NBO } & \text { normobaricoxygen } \\ \text { COPD } & \text { chronic pulmonary obstructive disease } \\ \text { HIV } & \text { Human Immunodeficiency Virus } \\ \text { AHR } & \text { adjusted hazard ratio } \\ \text { CI } & \text { confidence interval } \\ \text { CO } & \text { carbon monoxide } \\ \text { NPD } & \text { Nationwide Poisoning Database }\end{array}$




$\begin{array}{ll}\text { NHIRD } & \text { National Health Insurance Research Database } \\ \text { ICD-9-CM } & \text { International Classification of Diseases, Ninth Revision, Clinical Modification } \\ \mathrm{COHb} & \text { carboxyhemoglobin } \\ \mathrm{IRR} & \text { incidence rate ratio } \\ \text { ATA } & \text { atmosphere absolute }\end{array}$

\section{References}

1. United States Environmental Protection Agency. An Introduction to Indoor Air Quality (IAQ). Carbon Monoxide (CO). Available online: http:/ / www.epa.gov/iaq/co.html (accessed on 22 March 2016).

2. CDC (Centers for Disease Control and Prevention). Unintentional, non-fire-related, carbon monoxide exposures-United States, 2001-2003. MMWR Morb. Morta.l Wkly. Rep. 2005, 54, 36-39.

3. Prockop, L.D.; Chichkova, R.I. Carbon monoxide intoxication: An updated review. J. Neurol. Sci. 2007, 262, 122-130. [CrossRef] [PubMed]

4. Ernst, A.; Zibrak, J.D. Carbon monoxide poisoning. N. Engl. J. Med. 1998, 339, 1603-1608. [CrossRef] [PubMed]

5. Hampson, N.B.; Weaver, L.K. Carbon monoxide poisoning: A new incidence for an old disease. Undersea Hyperb. Med. 2007, 34, 163-168. [PubMed]

6. Hampson, N.B.; Piantadosi, C.A.; Thom, S.R.; Weaver, L.K. Practice recommendations in the diagnosis, management, and prevention of carbon monoxide poisoning. Am. J. Respir. Crit. Care 2012, 186, 1095-1101. [CrossRef] [PubMed]

7. Pan, Y.J.; Liao, S.C.; Lee, M.B. Suicide by charcoal burning in Taiwan, 1995-2006. J. Affect. Disord. 2010, 120, 254-257. [CrossRef] [PubMed]

8. Wolf, S.J.; Lavonas, E.J.; Sloan, E.P.; Jagoda, A.S. Clinical policy: Critical issues in the management of adult patients presenting to the emergency department with acute carbon monoxide poisoning. Ann. Emerg. Med. 2008, 51, 138-152. [CrossRef] [PubMed]

9. Buckley, N.A.; Juurlink, D.N.; Isbister, G.; Bennett, M.H.; Lavonas, E.J. Hyperbaric oxygen for carbon monoxide poisoning. Cochrane Database Syst. Rev. 2011, 4. [CrossRef] [PubMed]

10. Buckley, N.A.; Juurlink, D.N. Carbon monoxide treatment guidelines must acknowledge the limitations of the existing evidence. Am. J. Respir. Crit. Care 2013, 187, 1390. [CrossRef] [PubMed]

11. Hampson, N.B.; Piantadosi, C.A.; Thom, S.R.; Weaver, L.K. Reply: Carbon monoxide treatment guidelines must acknowledge the limitations of the existing evidence. Am. J. Respir. Crit. Care 2013, 187, 1390-1391. [CrossRef] [PubMed]

12. Weaver, L.K.; Hopkins, R.O.; Chan, K.J.; Churchill, S.; Elliott, C.G.; Clemmer, T.P.; Orme, J.F.; Thomas, F.O.; Morris, A.H. Hyperbaric oxygen for acute carbon monoxide poisoning. N. Engl. J. Med. 2002, 347, 1057-1067. [CrossRef] [PubMed]

13. Huang, C.C.; Ho, C.H.; Chen, Y.C.; Lin, H.J.; Hsu, C.C.; Wang, J.J.; Su, S.B.; Guo, H.R. Hyperbaric oxygen therapy is associated with lower short- and long-term mortality in patients with carbon monoxide poisoning. Chest 2017, 152, 943-953. [CrossRef] [PubMed]

14. National Health Insurance Research Database. Available online: http://nhird.nhri.org.tw/en/index.html (accessed on 22 April 2017).

15. Weaver, L.K. Carbon monoxide poisoning. N. Engl. J. Med. 2009, 360, 1217-1225. [CrossRef] [PubMed]

16. Buckley, N.A.; Isbister, G.K.; Stokes, B.; Juurlink, D.N. Hyperbaric oxygen for carbon monoxide poisoning: A systematic review and critical analysis of the evidence. Toxicol. Rev. 2005, 24, 75-92. [CrossRef] [PubMed]

17. Lin, J.-N.; Lin, C.-L.; Lin, M.-C.; Lai, C.-H.; Lin, H.-H.; Yang, C.-H.; Sung, F.-C.; Kao, C.-H. Risk of leukaemia in children infected with enterovirus: A nationwide, retrospective, population-based, Taiwanese-registry, cohort study. Lancet Oncol. 2015, 16, 1335-1343. [CrossRef]

18. Lee, C.-C.; Lee, M.-T.; Chen, Y.-S.; Lee, S.-H.; Chen, Y.-S.; Chen, S.-C.; Chang, S.-C. Risk of Aortic Dissection and Aortic Aneurysm in Patients Taking Oral Fluoroquinolone. JAMA Intern. Med. 2015, 175, 1839-1847. [CrossRef] [PubMed]

19. National Health Insurance Administration, Ministry of Health and Welfare. Items for Medical Payments. Available online: http:/ / www.nhi.gov.tw/query/query2.aspx (accessed on 23 April 2017). 
20. National Health Insurance Administration, Ministry of Health and Welfare. Classification of Diseases. Available online: http:/ / www.nhi.gov.tw/webdata/webdata.aspx?menu=18\&menu_id=703\&webdata_ id=1008 (accessed on 23 April 2017).

21. Annane, D.; Chadda, K.; Gajdos, P.; Jars-Guincestre, M.C.; Chevret, S.; Raphael, J.C. Hyperbaric oxygen therapy for acute domestic carbon monoxide poisoning: Two randomized controlled trials. Intens. Care Med. 2011, 37, 486-492. [CrossRef] [PubMed]

22. Scheinkestel, C.D.; Bailey, M.; Myles, P.S.; Jones, K.; Cooper, D.J.; Millar, I.L.; Tuxen, D.V. Hyperbaric or normobaric oxygen for acute carbon monoxide poisoning: A randomised controlled clinical trial. Med. J. Aust. 1999, 170, 203-210. [PubMed]

23. Huang, C.-C.; Chung, M.-H.; Weng, S.-F.; Chien, C.-C.; Lin, S.-J.; Lin, H.-J.; Guo, H.-R.; Su, S.-B.; Hsu, C.-C.; Juan, C.-W. Long-term prognosis of patients with carbon monoxide poisoning: A nationwide cohort study. PLoS ONE 2014, 9. [CrossRef] [PubMed]

24. Thom, S.R.; Taber, R.L.; Mendiguren, I.I.; Clark, J.M.; Hardy, H.M.; Fisher, A.B. Delayed neuropsychologic sequelae after carbon monoxide poisoning: Prevention by treatment with hyperbaric oxygen. Ann. Emerg. Med. 1995, 25, 474-480. [CrossRef]

25. Thom, S.R. Hyperbaric-oxygen therapy for acute carbon monoxide poisoning. N. Engl. J. Med. 2002, 347, 1105-1106. [CrossRef] [PubMed]

26. Weaver, L.K.; Valentine, K.J.; Hopkins, R.O. Carbon monoxide poisoning: Risk factors for cognitive sequelae and the role of hyperbaric oxygen. Am. J. Respir. Crit. Care 2007, 176, 491-497. [CrossRef] [PubMed]

27. Zou, J.-F.; Guo, Q.; Shao, H.; Li, B.; Du, Y.X.; Liu, M.-F.; Liu, F.-L.; Dai, L.-X.; Lin, H.-J.; Su, S.-B.; et al. Lack of pupil reflex and loss of consciousness predict 30-day neurological sequelae in patients with carbon monoxide poisoning. PLOS ONE 2015, 10. [CrossRef] [PubMed]

28. Zou, J.-F.; Guo, Q.; Shao, H.; Li, B.; Du, Y.-X.; Liu, M.-F.; Liu, F.-L.; Dai, L.-X.; Chung, M.-H.; Lin, H.-J. A positive Babinski reflex predicts delayed neuropsychiatric sequelae in Chinese patients with carbon monoxide poisoning. Biomed. Res. Int. 2014, 2014, 1-5. [CrossRef] [PubMed]

29. Hu, H.; Pan, X.; Wan, Y.; Zhang, Q.; Liang, W. Factors affecting the prognosis of patients with delayed encephalopathy after acute carbon monoxide poisoning. Am. J. Emerg. Med. 2011, 29, 261-264. [CrossRef] [PubMed]

30. Shen, C.-H.; Lin, J.-Y.; Pan, K.-T.; Chou, Y.-C.; Peng, C.-K.; Huang, K.-L. Predicting poor outcome in patients with intentional carbon monoxide poisoning and acute respiratory failure: A retrospective study. J. Med. Sci. 2015, 35, 105-110. [CrossRef]

31. Liu, K.Y.; Beautrais, A.; Caine, E.; Chan, K.; Chao, A.; Conwell, Y.; Law, C.; Lee, D.; Li, P.; Yip, P. Charcoal burning suicides in Hong Kong and urban Taiwan: An illustration of the impact of a novel suicide method on overall regional rates. J. Epidemiol. Commun. Health 2007, 61, 248-253. [CrossRef] [PubMed]

32. Runyan, C.W.; Johnson, R.M.; Yang, J.; Waller, A.E.; Perkis, D.; Marshall, S.W.; Coyne-Beasley, T.; MSPH, K.S.M. Risk and protective factors for fires, burns, and carbon monoxide poisoning in U.S. households. Am. J. Prev. Med. 2005, 28, 102-108. [CrossRef] [PubMed]

33. Johnson-Arbor, K.; Liebman, D.L.; Carter, E.M. A survey of residential carbon monoxide detector utilization among Connecticut Emergency Department patients. Clin. Toxicol. 2012, 50, 384-389. [CrossRef] [PubMed]

34. Lemstra, M.; Rogers, M.; Moraros, J. Income and heart disease: Neglected risk factor. Can. Fam. Physician 2015, 61, 698-704. [PubMed]

35. Behera, S.K.; Winkleby, M.A.; Collins, R. Low awareness of cardiovascular disease risk among low-income African-American women. Am. J. Health Promot. 2000, 14, 301-305. [CrossRef] [PubMed]

(C) 2018 by the authors. Licensee MDPI, Basel, Switzerland. This article is an open access article distributed under the terms and conditions of the Creative Commons Attribution (CC BY) license (http://creativecommons.org/licenses/by/4.0/). 